\title{
THE PROGNOSTIC SIGNIFICANCE OF TUMOR CELL DETECTION IN INTRAOPERATIVE PLEURAL LAVAGE AND LUNG TISSUE CULTURES FOR PATIENTS WITH LUNG CANCER
}

\author{
J. Buhr, MD \\ K. H. Berghäuser, $\mathrm{MD}^{\mathrm{b}}$ \\ S. Gonner, $\mathrm{MD}^{\mathrm{b}}$ \\ C. Kelm, MD ${ }^{\mathrm{a}}$ \\ E. A. Burkhardt, $\mathrm{PhD}^{\mathrm{c}}$ \\ W. M. Padberg, MD
}

\begin{abstract}
Methods: Three hundred forty-two patients with lung cancer and 99 patients with nonneoplastic lung diseases (control group) underwent intraoperative pleural lavage with $300 \mathrm{ml}$ physiologic saline solution before (lavage I) and after resection (lavage II). Results: Studies of the lavage fluid in all control patients were negative, that is, there were no false positive findings. Tumor cells were found in lavage $I$ in 132 patients $(38.6 \%)$ and also in lavage $I I$ in 99 of them. In stage I (pT1 N0, pT2 N0) lung cancer, tumor cell detection was possible in 47 patients $(28.6 \%)$. The 4-year survival of patients with resected non-small-cell lung cancer was $24 \%$ (95\% confidence interval, $16 \%$ to $32 \%)$ if lavage I results were positive and $52 \%$ (95\% confidence interval, $45 \%$ to $59 \%$ ) if lavage I results were negative (all stages, $p=0.007$ ). For patients with stage I disease $(n=164)$ the 4-year survival was $35 \%(95 \%$ confidence interval, $18 \%$ to $52 \%$ ) if lavage I results were positive $(n=47)$, and $69 \%(95 \%$ confidence interval, $60 \%$ to $78 \%)$ if lavage $I$ results were negative $(n=117)(p=0.037)$. On multivariate analysis the positive cytologic result in intraoperative pleural lavage was an additional prognostic factor for our patients. To prove how the tumor cells enter the pleural cavity, we performed tissue cultures of tumor-free parenchyma in 23 cases of lung cancer. Tumor cell detection by histology and immunohistology was possible in 16 cases (69.6\%). Detection of tumor cells in pleural lavage fluid before resection proves that tumor cells have spread into the pleural cavity. Conclusion: The positive result in pleural lavage seems to be a prognostic predictor for patients with lung cancer. (J Thorac Cardiovasc Surg 1997; 113:683-90)
\end{abstract}

n n 1958 Spjut and associates ${ }^{1}$ reported the results of pleural cavity washings on 49 patients undergoing thoracotomy for lung cancer. They presented the cytologic examination of pleural lavage. In 16 of their patients $(32.7 \%)$ the cytologic result was positive for malignant cells. In 1984 Eagan and col-

From the Department of General and Thoracic Surgery, ${ }^{\mathrm{a}}$ Institute for Pathology, ${ }^{b}$ and Tumor Center ${ }^{c}$ (Department of Statistics), Justus Liebig University, D-35392 Giessen, Germany.

Read at the Twenty-second Annual Meeting of The Western Thoracic Surgical Association, Maui, Hawaii, June 26-29, 1996.

Received for publication July 3, 1996; revisions requested July 29, 1996; revisions received Dec. 2, 1996; accepted for publication Dec. 6, 1996.

Address for reprints: J. Buhr, MD, Department of General and Thoracic Surgery, Justus Liebig University, Rudolf-Buchheim-Str. 7, D-35392 Giessen, Germany.

Copyright (c) 1997 by Mosby-Year Book, Inc.

$0022-5223 / 97 \$ 5.00+0 \quad 12 / 6 / 79790$ leagues $^{2}$ followed with their results with pleural lavage cytology after pulmonary resection for lung cancer. The cytologic results of lavage were positive for malignant cells in 12 of their 135 patients $(8.9 \%)$. In both studies intraoperative pleural lavage was performed only after pulmonary resection for lung cancer, and neither study considered its prognostic value.

In 1989 Kondo and coworkers ${ }^{3}$ reported on the prognostic factor of positive cytologic results in pleural lavage immediately after thoracotomy for patients with lung cancer. At the same time, we $\mathrm{w}^{4}$ published our first preliminary report about the positive cytologic results in intraoperative pleural lavage after opening the chest and before resection. At that time we found a significantly poorer 2-year survival for patients with positive findings in pleural lavage.

Now we present the results of our advanced prospective study about intraoperative pleural lavage in lung cancer. To find out how the tumor cells 
Table I. Characteristics of the patients, operations, histologic type, and final tumor stage of the patients undergoing intraoperative pleural lavage $(n=342)$

\begin{tabular}{lrc}
\hline & No. & $\%$ \\
\hline Sex & 294 & 86 \\
Male & 48 & 14 \\
Female & 109 & 31.9 \\
Operations & 27 & 7.9 \\
Pneumoncctomies & 185 & 54.1 \\
Bilobectomies & 15 & 4.4 \\
Lobectomies & 6 & 1.7 \\
Wedge resections & & \\
Exploratory thoracotomies & 164 & 47.9 \\
Postoperative tumor stage & 55 & 16.1 \\
Stage I & 75 & 21.9 \\
Stage II & 25 & 7.3 \\
Stage IIIA & 23 & 6.7 \\
Stage IIIB & & \\
Stage IV & 189 & 55.3 \\
Histologic type & 102 & 29.8 \\
Squamous cell carcinomas & 22 & 6.4 \\
Adenocarcinomas & 17 & 5 \\
Adenosquamous carcinomas & 12 & 3.5 \\
Large-cell carcinomas &
\end{tabular}

enter pleural cavity before pulmonary resection, we performed lung tissue cultures of the surrounding tumor-free lung parenchyma.

\section{Patients and methods}

Intraoperative pleural lavage. From January 1986 to December 1987 and from January 1990 to December 1995, 484 patients underwent resection for primary lung cancer at the Department of General and Thoracic Surgery of the Justus Liebig University at Giessen. Intraoperative pleural lavage was performed in 342 patients (study group) with the first manifestation of lung cancer (294 men and 48 women, average age 58.9 years). We performed pleural lavage in the time from January 1986 to December 1987 as a experimental evaluation. After obtaining our results with the significantly poorer 2-year survival for those patients with a positive result of intraoperative pleural lavage, ${ }^{4}$ we continued our study in January 1990 . Patients who had previously received chemotherapy or radiotherapy and patients with a malignant secondary tumor were excluded. Preoperative evaluation included a detailed history and physical examination, biochemical profile, chest x-ray examination, bronchoscopy, computed tomography of the chest and upper portion of the abdomen, and abdominal ultrasonographic scan. Neither preoperative computed tomographic scan of the brain nor routine cervical or anterior mediastinoscopy was performed in the absence of clinical evidence of brain involvement and in the presence of a negative mediastinal window shown by tomographic examination, respectively. None of the patients had an intraoperative pleural effu- sion. Intraoperative pleural lavage was performed in every possible case as described herein and without patient consent, because this examination did not influence our intraoperative procedures and postoperative management.

The control group comprised 99 patients with nodules of the lung, which on postoperative histologic examination were not malignant. In both groups we performed intraoperative pleural lavage in the same manner.

After an anterolateral thoracotomy, the pleural cavity was filled with $300 \mathrm{ml}$ physiologic saline solution before any further manipulation of the pulmonary parenchyma. To obtain only desquamated cells, we avoided touching the pleural surfaces. The surgeon carefully washed the pleural cavity by hand for 1 minute. The fluid was then suctioned off, and 1000 units of heparin and $50 \mathrm{ml}$ of Ebner's solution ${ }^{4,5}$ were added (lavage I). After surgical resection of the tumor and lymph node dissection, the washing was repeated in the same manner (lavage II). Immediately after the lavages, the fluid was shaken, and 12 cytocentrifugates of $2 \mathrm{ml}$ each $(24 \mathrm{ml})$ were prepared and stained according to the Papanicolaou method. From the remaining lavage fluid, the sediment was separated by centrifugation. After erythrocytolysis with acetic acid and fixation in alcohol, the sediments were embedded in paraffin, completely serial-sectioned, and stained with hematoxylin and eosin. Microscopic examination was performed by two physicians (J.B., K.H.B.) and their findings reported according to Papanicolaou's classification. The results of the cytologic examination were divided into two categories: "negative lavage" and "positive lavage." Papanicolaou classes I to III were declared as "negative lavage" and Papanicolaou classes IV to $\mathrm{V}$ as "positive lavage."

The primary tumor and lymph node status was classified according to the international staging system reported by Mountain. ${ }^{6}$ Careful intraoperative staging at the time of surgical resection was done by dissecting intrapulmonary and hilar nodes and sampling mediastinal lymph node stations. The histologic type of the tumor was determined by applying the World Health Organization classification. The results of the histopathologic examination regarding the status of lymph node metastasis and pleural involvement by the tumor were documented. ${ }^{8}$

The $\chi^{2}$ test was used to evaluate the significance of the relationship between the cytologic result of intraoperative pleural lavage and the histopathologic findings. Survival curves were calculated according to the method of Kaplan and Meier ${ }^{9}$ and the log-rank test was used to compare the survival curves. ${ }^{10}$ Perioperative mortality $(17 / 342,4.9 \%)$ was included in the calculation of the survival. The survival was calculated without censored data, and patients who died with no evidence of recurrence and with an unrelated disease were not excluded. Cox's proportional hazards stepwise model was used for multivariate analysis. ${ }^{11}$ For all calculations we used the program SPSS (Statistical Package for the Social Sciences) for Windows (SPSS, Inc., Chicago, III.).

Lung tissue cultures. To clarify how the tumor cells enter the pleural space, we performed tissue cultures of tumor-free lung parenchyma surrounding the lung cancer. 


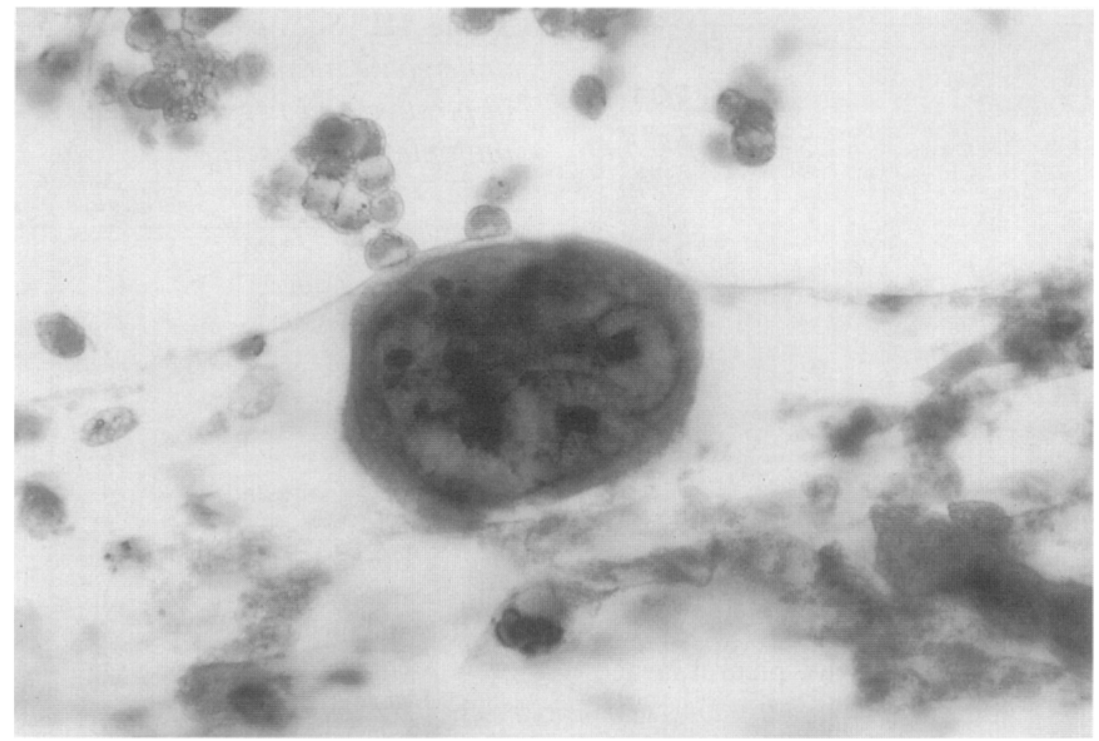

Fig. 1. A malignant cell in intraoperative pleural lavage fluid (lavage I) of a squamous cell carcinoma (pT2 N0, stage I, G2; Papanicolaou staining; original magnification $\times 500$ ).

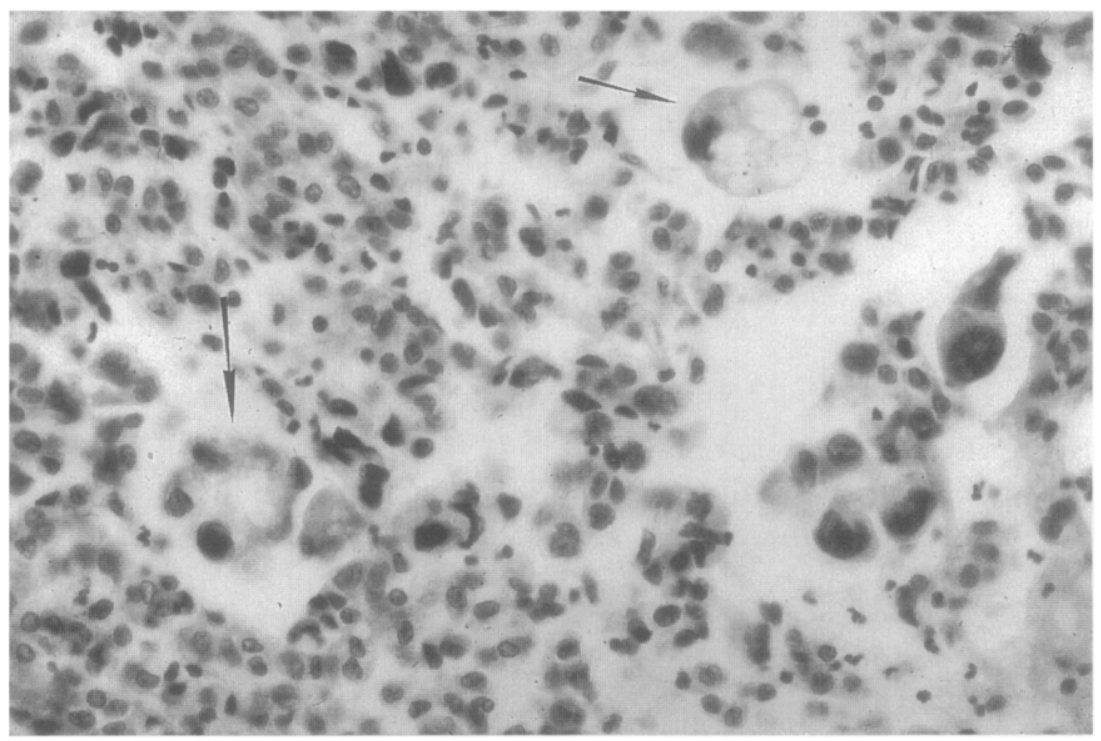

Fig. 2. Malignant cells in the sediment of intraoperative pleural lavage (lavage I, arrows) (adenocarcinoma, pT2 N0, stage I, G2-3; hematoxylin and eosin staining, original magnification $\times 500$ ).

In 23 unselected patients, operated on in June and July of 1994 (stage I, $n=5$; stage II, $n=7$; stage IIIA, $n=11$ ), we used lung parenchyma for tissue culture. The lung pieces were examined histologically (hematoxylin and eosin staining), and only tumor-free lung parenchyma ( 6 $\mathrm{cm}$ distant from the lung cancer) was taken for tissue cultures. Three-millimeter squares were washed and incubated in Häm's F12 medium and $12 \%$ fetal calf serum. Every third day a new incubation was done (Häm's F12 medium and fetal calf serum). One or two pieces of lung parenchyma were selected for histologic (hematoxylin and eosin staining) examination.

\section{Results}

The characteristics of the patients, operations, histologic type, and final tumor stage of the patients undergoing intraoperative pleural lavage are shown. in Table I. 


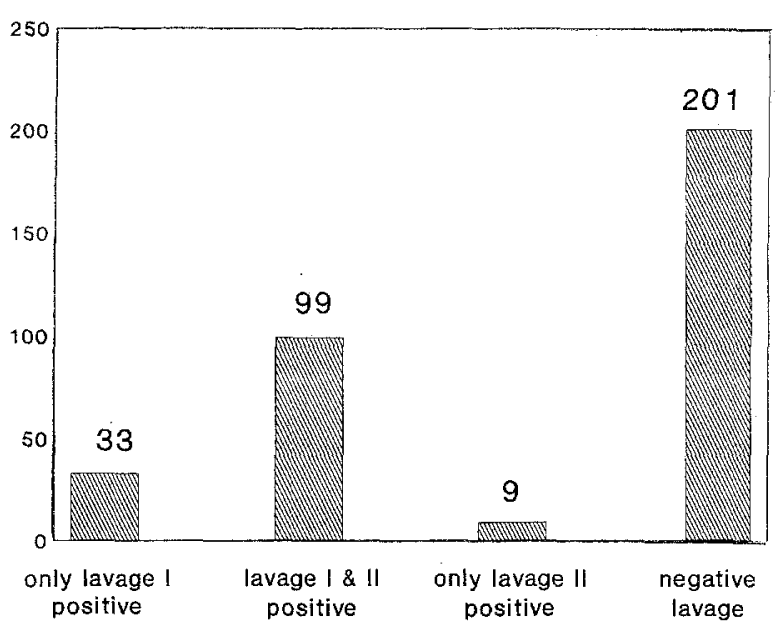

Fig. 3. Cytologic results in intraoperative pleural lavage ( $n=342$ patients).

Table II. Cytologic results in intraoperative pleural lavage according to histology of lung cancer ( $n=$ 342; $p=$ not significant)

\begin{tabular}{lrrrr} 
& & & \multicolumn{2}{c}{ Positive } \\
\cline { 4 - 5 } & Total & Negative & No. & $\%$ \\
\hline Squamous cell carcinoma & 189 & 113 & 76 & 40.2 \\
Adenocarcinoma & 102 & 59 & 43 & 42.1 \\
Adenosquamous carcinoma & 22 & 12 & 10 & 45.5 \\
Large-cell carcinoma & 17 & 10 & 7 & 41.2 \\
Small-cell carcinoma & 12 & 7 & 5 & 41.7 \\
\hline
\end{tabular}

Control group. No positive results were obtained cytologically in any of the 99 patients with nonmalignant disease of the lung; that is, there were no false positive findings.

Study group. Of the 342 patients with lung cancer in whom pleural lavage was performed, $132(38.6 \%)$ had positive results in lavage I (Figs. 1 and 2); 99 of them also had positive findings in lavage II. In nine patients $(2.6 \%)$ only lavage II was positive. In most of these nine patients in whom only lavage II was positive, adhesions of the lung with the parietal pleura were found. All 141 patients (41.2\%) in whom cytologic tumor cell detection was possible were assigned to the "positive lavage" group (Fig. 3) for the calculations of "stage" and "histologic type." To calculate the survivals, we used only the data of the 132 patients with a positive lavage I. The nine patients with a positive lavage II were excluded from the survival analyses, to avoid the effect of any possible tumor dissemination by surgery. However,
Table III. Sites of recurrences for patients undergoing intraoperative pleural lavage (subdivided in positive and negative lavage $1 ; 43$ patients with multiple metastases)

\begin{tabular}{lrrr}
\hline & Negative & Positive & $p$ Value \\
\hline All stages $(n=342)$ & & & \\
Local recurrence & 12 & 18 & $p=0.69$ \\
Distant metastasis & 23 & 78 & $p=0.008$ \\
Contralateral lung & 4 & 22 & \\
Liver & 9 & 23 & \\
Brain & 3 & 19 & \\
Kidney & 5 & 4 & \\
Suprarenal gland & 4 & 17 & \\
Others & 6 & 33 & \\
Stage I $(n=161)$ & & & \\
Local recurrence & 1 & 3 & $p=0.85$ \\
Distant metastasis & 7 & 13 & $p=0.039$ \\
Contralateral lung & 1 & 2 & \\
Liver & 2 & 7 & \\
Brain & 2 & 3 & \\
Kidney & 1 & 1 & \\
Suprarenal gland & 0 & 0 & \\
Others & 1 & 4 & \\
& & &
\end{tabular}

the number of patients with a positive lavage II was too small to make any statistical analysis.

Stage. Malignant cells were detected in 47 of 164 patients with stage I disease $(28.6 \%), 20$ of 55 with stage II disease (36.4\%), 59 of 100 with stage III disease $(59 \%)$, and 15 of 23 with stage IV disease $(65.2 \%)$. Positive findings in intraoperative pleural lavage fluid were more frequent in advanced tumor stages ( $p=0.0001$ in stage I versus stages II to IV).

Histology. We found no association between the histologic classification of the primary tumor and cytologic findings of the intraoperative pleural lavage. The rate of positive results was nearly equal in all histologic types (Table II).

Sites of recurrences. We found a significantly higher rate of distant metastases in the patients who had a positive pleural lavage I as opposed to those with a negative lavage: we found distant metastases in 78 of the 132 patients (all stages) with a positive lavage I. Of the 201 patients (all stages) with a negative lavage I, distant metastases were detected in only $23(p=$ 0.008). In stage I lung cancer we found distant metastases in 13 of the 47 patients with a positive lavage I and in seven of the 114 patients with a negative lavage I $(p=0.039)$ (Table III).

Prognosis. Median follow-up for all patients was 46 months. The 4-year survival of patients undergoing curative resection of non-small-cell lung cancer 


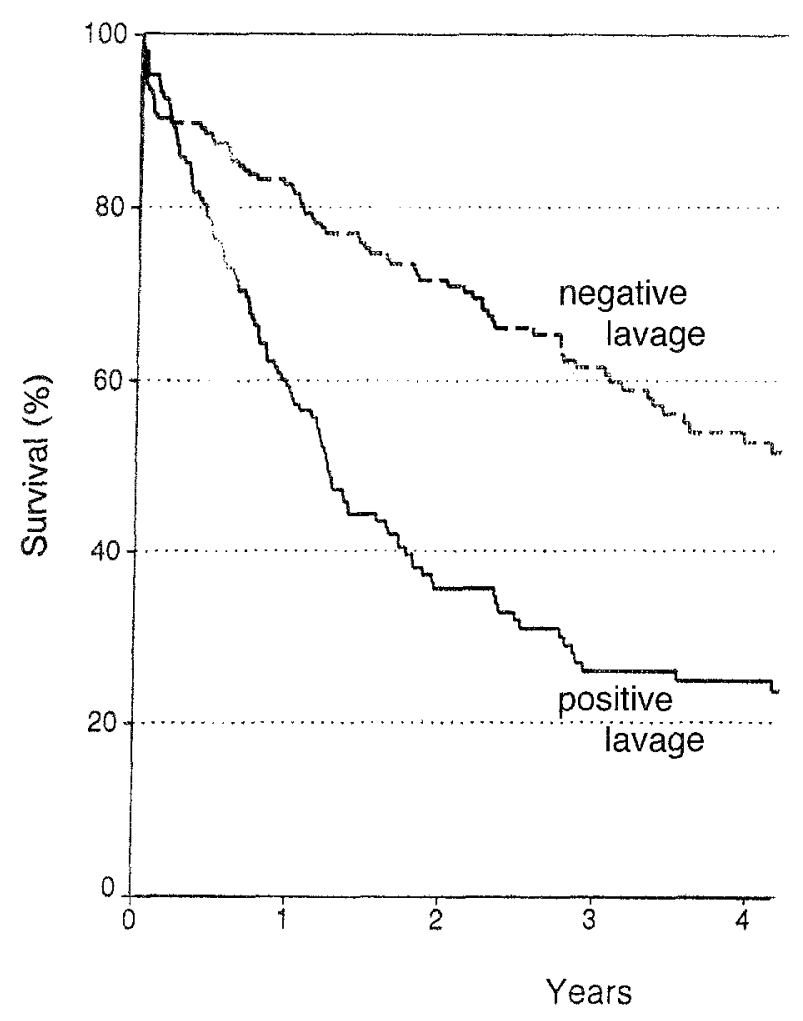

Fig. 4. Four-year survival of all patients undergoing intraoperative pleural lavage, subdivided into "positive lavage" $(n=132)$ and "negative lavage" $(n=201)$ groups $(p=$ $0.007)$.

was $24 \%$ ( $95 \%$ confidence interval, $16 \%$ to $32 \%$ ) if lavage I was positive and $52 \%$ (95\% confidence interval, $45 \%$ to $59 \%$ ) if lavage I was negative (all stages, $p=0.007$ ) (Fig. 4). If the 4-year survival for patients with non-small-cell lung cancer was calculated on the basis of positive and negative pleural lavage findings, the following results were obtained: the cumulative 5-year survival for patients undergoing curative resection for non-small-cell lung cancer in stage I (pT1 N0, pT2 N0) $(n=164)$ was $29.1 \%$ if lavage was positive $(n=47)$ and $69.8 \%$ if lavage was negative $(n=117)(p=0.037)$ (Fig. 5). The survivals and $95 \%$ confidence intervals of all patients are presented in Table IV.

Multivariate analysis. By multivariate analysis of the data on our patients undergoing intraoperative pleural lavage, we found that a positive cytologic result was an independent risk factor for death (relative risk $0.6017 ; 95 \% p$ value 0.0001 ). Only the final tumor stage and the age of the patients were also prognostic factors. The relative risk of a poorer

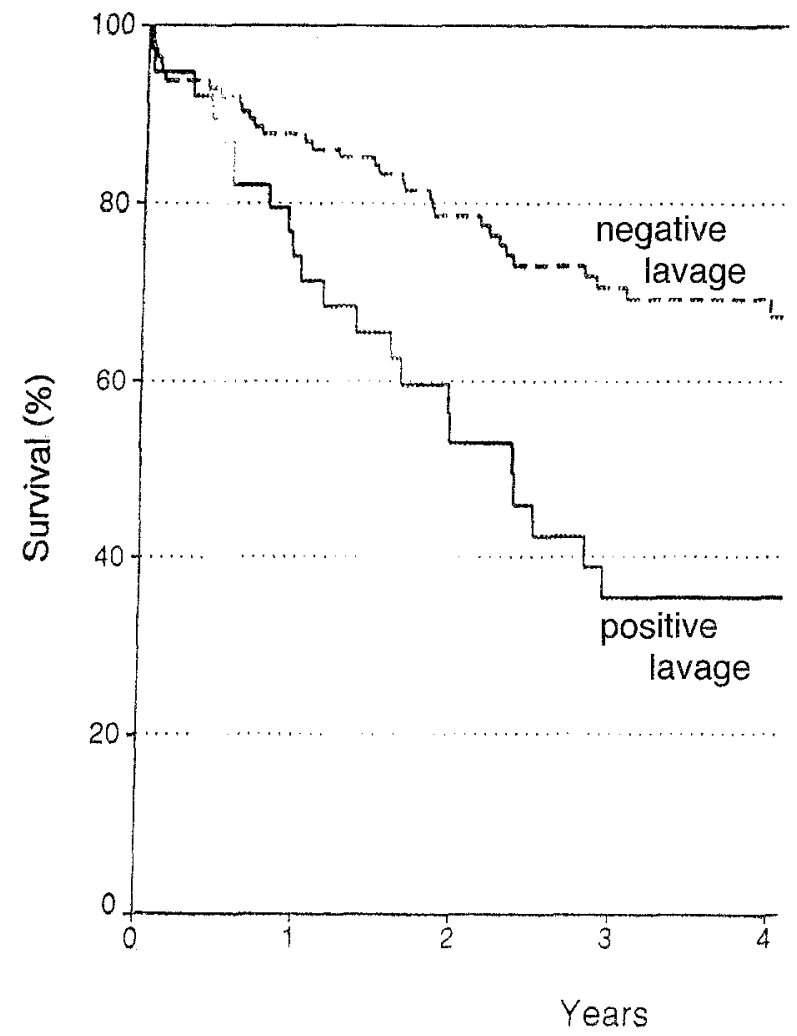

Fig. 5. Four-year survival of patients undergoing curative resection for stage $I$ lung cancer, divided into "positive lavage" ( $n=47)$ and "negative lavage" $(n=114)$ groups, if lavage I (before resection) was positive ( $p=$ $0.037)$.

prognosis was 1.8252 (95\% confidence interval 1.3430 to 2.4804 ).

Lung tissue cultures. In 16 patients $(69.6 \%)$ we detected tumor cells in lung parenchyma between the third and thirty-sixth days (median 15 days) after incubation. In all patients tumor cells were found in lymphatic vessels (Fig. 6). In 14 of these patients the intraoperative pleural lavage was also positive $(87.5 \%)$. We found tumor cells in only two lung tissue cultures when the pleural lavage of the same patient was negative.

\section{Discussion}

The TNM classification of malignant tumors was revised in 1992 and a new edition is in progress. The pTNM classification of lung cancer is mostly based on the histologic examination of the tumor. ${ }^{6}$ The diagnostic procedures of the pleural cavity are still insufficient. Only malignant pleural effusion is included in the TNM classification as a T4 spread of 


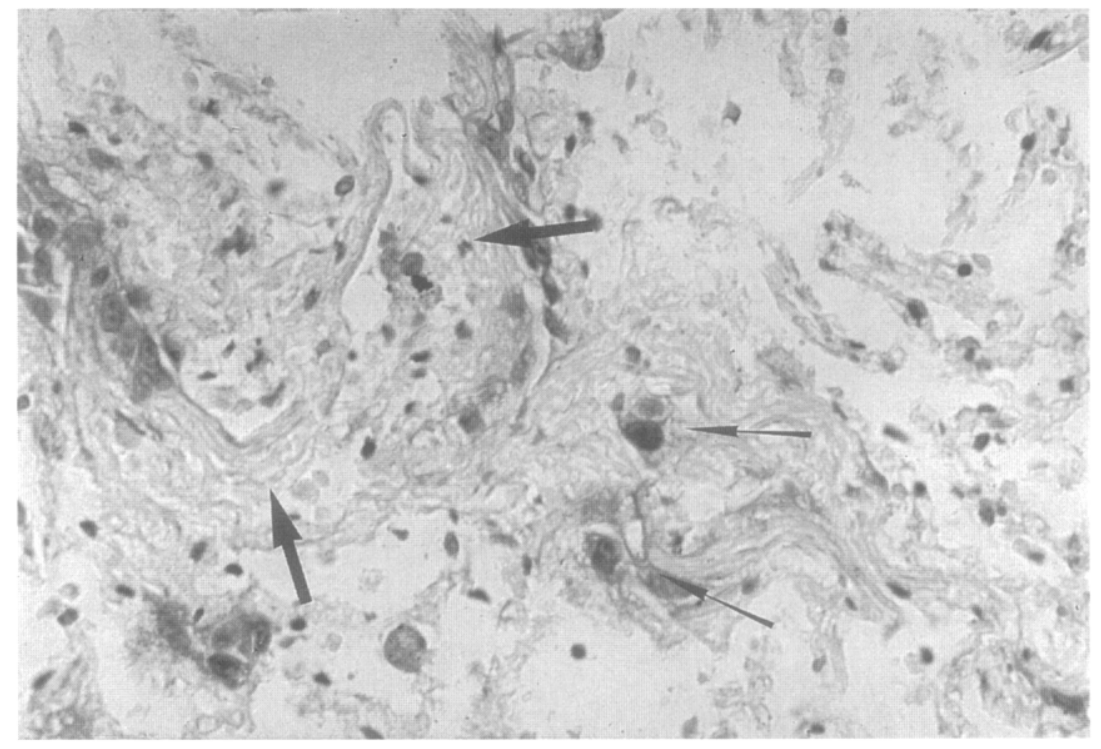

Fig. 6. Tumor cells (small arrows) in lung tissue culture in a lymphatic vessel near a small pulmonary artery and vein (big arrows) 9 days after incubation of tumor-free lung parenchyma of an adenocarcinoma of the lung (pT2 N0; hematoxylin and eosin staining, original magnification $\times 400$ ).

Table IV. Four-year survivals of 342 patients undergoing intraoperative pleural lavage, subdivided into positive and negative lavage I (including 95\% confidence intervals)

\begin{tabular}{|c|c|c|c|c|c|}
\hline & $n$ & $1 y r$ & $2 y r$ & $3 y r$ & $4 y r$ \\
\hline \multicolumn{6}{|l|}{ All } \\
\hline Positive & 132 & $59(51$ to 67$)$ & $36(28$ to 44$)$ & 26 (18 to 34$)$ & 24 (16 to 32$)$ \\
\hline Negative & 201 & $82(76$ to 88$)$ & $72(65$ to 79$)$ & $61(53$ to 69$)$ & $52(44$ to 60$)$ \\
\hline \multicolumn{6}{|l|}{$p=0.007$} \\
\hline \multicolumn{6}{|l|}{ Stage I } \\
\hline Positive & 47 & $71(56$ to 86$)$ & $53(36$ to 70$)$ & $36(19$ to 53$)$ & $35(18$ to 52$)$ \\
\hline Negative & 114 & 87 (79 to 93$)$ & $79(71$ to 87$)$ & $71(62$ to 80$)$ & $69(60$ to 78$)$ \\
\hline \multicolumn{6}{|l|}{$p=0.037$} \\
\hline \multicolumn{6}{|l|}{ Stage II } \\
\hline Positive & 20 & $69(51$ to 87$)$ & $34(15$ to 53$)$ & $28(11$ to 47$)$ & $24(5$ to 43$)$ \\
\hline Negative & 33 & 85 (71 to 99$)$ & 74 (53 to 91$)$ & $58(37$ to 79$)$ & 48 ( 23 to 73$)$ \\
\hline \multicolumn{6}{|l|}{$p=0.076$} \\
\hline \multicolumn{6}{|l|}{ Stage IIIA } \\
\hline Positive & 37 & $57(43$ to 71$)$ & $30(16$ to 44$)$ & $18(5$ to 31$)$ & $10(0$ to 20$)$ \\
\hline Negative & 31 & 77 (62 to 92$)$ & $66(48$ to 84$)$ & $50(31$ to 69$)$ & $36(17$ to 55$)$ \\
\hline$p=0.042$ & & & & & \\
\hline
\end{tabular}

lung cancer. The criterion for malignant pleural effusion is the cytologic detection of tumor cells. ${ }^{6}$ In these patients a curative resection cannot be performed.

We present the results of a prospective study, in which pleural lavage was done after the chest had been opened to determine whether tumor cell dissemination into the pleural cavity had already occurred in the early stages of lung cancer. A second lavage after resection of lung cancer and lymph node dissection showed whether surgical manipula- tion of the lung parenchyma had led to tumor cell contamination of the pleural cavity.

Cytologic examination of peritoneal lavage fluid seems to be an important prognostic factor in malignant solid tumors of the abdomen. ${ }^{12-15}$ Spjut and associates $^{1}$ first described the results of cytologic examination of pleural cavity washings after pulmonary resection of lung cancer in 1958; Eagan and colleagues $^{2}$ followed in 1984. Their reports examined only the positive cytologic results of pleural lavage after surgical manipulation. The positive 
findings might occur as a result of the exfoliation and contamination of cancer cells into the pleural space during surgical manipulation. We must discriminate between pleural lavage performed immediately after thoracotomy and that performed after pulmonary resection. After publication of our preliminary reports about tumor cell findings in intraoperative pleural lavage fluid after opening of the chest, ${ }^{4,5}$ other authors likewise reported detecting tumor cells in intraoperative pleural lavage fluid. ${ }^{16-19}$ Up to now we have performed intraoperative pleural lavage in 342 patients with lung cancer. Our recent results emphasize the prognostic significance of positive findings of intraoperative pleural lavage for patients with lung cancer.

The detection of tumor cells in pleural lavage fluid before resection proves that tumor cells have spread into the pleural cavity, even in the early stages of lung cancer. The pleural cavity in patients with positive findings of intraoperative pleural lavage was not contaminated by the surgical intervention, because manipulation of the lung and the visceral pleura was excluded before the first lavage was done.

How the tumor cells enter the pleural cavity is still unknown. We believe that the most likely mode is spread via the lymphatic vessels. The results of tumor cell detection in lung parenchyma, which were initially tumor-free at the beginning of lung tissue cultures, could be explained by the tumor cell detection in the intraoperative pleural lavage fluid. In addition, however, it must be a result of exfoliation of cancer cells and may be possible without any pleural effusion. Preformed stomas that connect subpleural lymphatics with the pleural space could account for the tumor cell dissemination. $20-25$

The cytologic method of examination of the intraoperative pleural lavage fluid that we used is highly specific. We did not find any false positive results. We included Papanicolaou class IV and V cytologic results in our "positive lavage" group. This might explain why we had a higher percentage of positive cytologic findings in pleural lavage than other authors. 2, 3, 16-19 In most of the other studies, positive results in squamous cell carcinoma were not as high as in our study.

The positive findings of pleural lavage are a significant prognostic factor for patients with lung cancer. Our recent results with 342 patients who had lung cancer confirm our first results with 59 patients. We found a significantly poorer 4-year survival if tumor cell detection was possible. The survival was
$35 \%$ in stage I lung cancer if we found tumor cells in the lavage fluid and $69 \%$ if the result was negative. In our small group of patients with stage II disease the difference was not significant, but in stage IIIA the survival was statistically significantly worse if the results of pleural lavage were positive. In a multivariate analysis, a positive cytology of pleural lavage was an additional prognostic factor for patients with lung cancer.

Therefore, cytologic examination of intraoperative pleural lavage fluid should be done when assessing the final tumor stage in patients with lung cancer. A positive result should be added to the pTNM classification. In view of these findings, we have started a new randomized study of adjuvant chemotherapy for those patients with a positive cytology of intraoperative pleural lavage in stage I lung cancer.

\section{REFERENCES}

1. Spjut JH, Hendrix VJ, Ramirez GA, Roper CL. Carcinoma cells in pleural cavity washings. Cancer 1958;11:1222-5.

2. Eagan RT, Bernatz PE, Payne WS, et al. Pleural lavage after pulmonary resection for bronchogenic carcinoma. J Thorac Cardiovasc Surg 1984;88;1000-3.

3. Kondo $H$, Naruke $T$, Tsuchiya $R$, et al. Pleural lavage cytology after thoracotomy as a prognostic factor for patients with lung cancer. Jpn J Cancer Res 1989;80:233-7.

4. Buhr J, Berghäuser KH, Morr H, Dobroschke J. Determination of the prognosis of bronchogenic carcinoma by intraoperative pleural lavage. Deutsch Med Wochenschr 1989;114: 1597-601.

5. Buhr J, Berghäuser KH, Morr H, Dobroschke J, Ebner HJ. Tumor cells in intraoperative pleural lavage: an indicator for the poor prognosis in bronchogenic carcinoma. Cancer 1990; 65:1801-4.

6. Mountain CF. A new international staging system for lung cancer. Chest 1986;89(suppl):2255-335.

7. World Health Organization. Histological typing of lung tumors. 2nd ed., Geneva: World Health Organization, 1981.

8. Hermanek P, Gall FP. Grundlagen der klinischen Onkologie. Baden-Baden, Kö1n, New York: Witzstrock, 1979.

9. Kaplan EL, Meier P. Nonparametric estimation from incomplete observations. J Am Stat Assoc 1958;53:457-81.

10. Peto R, Pike MC, Armitage P, et al. Design and analysis of randomized clinical trials requiring prolonged observation of each patient. Br J Cancer 1977;35:1-39.

11. Cox DR. Regression models and life tables. J R Stat Soc B 1972;34:187-220.

12. Martin JK, Goelnner JR. Abdominal fluid cytology in patients with gastrointestinal malignant lesions. Mayo Clin Proc 1986;61:467-71.

13. Moore GE, Sako K, Kondo T, Badillo J, Burke E. Assessment of the exfoliation of tumor cells into the body cavities. Surg Gynecol Obstet 1961;112:469-74.

14. Nakajima T, Harashima S, Hirata M, Kajitani T. Prognostic and therapeutic value of peritoneal cytology in gastric cancer. Acta Cytol 1978;22:225-9. 
15. Warshaw AL. Implications of peritoneal cytology for staging of early pancreatic cancer. Am J Surg 1991;161:26-32.

16. Hsu JY, Chen CY, Huang CM, Chiang CD. Intraoperative pleural lavage in lung cancer patients. J Formos Med Assoc 1992;91:47-51.

17. Kondo H, Asamura H, Suemasu K, et al. Prognostic significance of pleural lavage cytology immediately after thoracotomy in patients with lung cancer. J Thorac Cardiovasc Surg 1993;106:1092-7.

18. Okada M, Tsubota N, Yoshimura M, et al. Cytology of pleural effusion and lavage samples at thoracotomy in cases of primary lung cancer. Haigan 1992;32:45-52.

19. Okumura M, Ohshima S, Kotake $Y$, et al. Intraoperative pleural lavage cytology in lung cancer patients. Ann Thorac Surg 1991;51:599-604.

20. Black LF. The pleural space and pleural fluid. Mayo Clin Proc 1972;493-506.

21. Jones JPS, editor. Pathology of the mesothelium. London: Springer, 1987.

22. Kanazawa $\mathrm{K}$. Exchange through the pleura. In: Chretien $\mathbf{J}$, Bigmon J, Hirsch A, editors. The pleura in health and disease. New York: Marcel Dekker, 1985:195-231.

23. Tobin CE. Human pulmonic lymphatics. Anat Rec 1957;127: 611-33.

24. Wang NS. The performed stomas connecting the pleural cavity and the lymphatics in the parietal pleura. Am Rev Respir Dis 1975;111:12-20.

25. Zeidman I, Buss JM. Experimental studies on the spread of cancer in the lymphatic system. IV. Retrograde spread. Cancer Res 1959;19:1114-7.

\section{Discussion}

Dr. Walter B. Cannon (Palo Alto, Calif.). This paper compares the differences between pathologic stage of disease versus clinical stage. As Dr. Buhr has said, the most important patients that they are dealing with are those with stage I disease-patients who are thought to have been cured because the tumor is out and because lymph nodes are not involved. He, however, has found that in numerous patients there is a significant involvement of the pleural cavity by tumor cells. The practice of evaluating cytologic characteristics of fluid within a cavity has long been in effect in the treatment of ovarian cancer. Peritoneal lavages looking at the fluid after the procedure have altered dramatically how these patients are treated and certainly has altered the prognosis-from a $70 \%$ 4-year survival to a 35\% 4-year survival if the pleural cavity is contaminated by tumor cells. The issue that I raise here concerns the situation in which the recurrences of these tumors occur in patients with only pleural involvement. Is the recurrence in the pleura or is it systemic recurrence? If it is a pleural recurrence, should local treatment factors be taken into account at the time of an operation, such as installation of chemotherapeutic agents within the pleural cavity or possibly even installation of sterile water into the pleural cavity? Can you answer these questions for us, Dr. Buhr?

Dr. Buhr. We found that local recurrence is no more common in patients with a positive result on pleural lavage than in those with a negative result. Those patients died with metastases, so we think this was only a sign that tumor had spread in the whole body, not only in the chest. We use systemic chemotherapy for patients who have metastases. We do not instill any chemotherapy or water in the pleural cavity after the resection.

Dr. Cannon. Why are these patients with apparent T4 disease having as high as a 35\% 4-year survival compared with those patients who would have pleural fluid with positive cytology, who would have a lesser 4-year survival?

Dr. Buhr. I do not think the positive cytologic findings in pleural lavage fluid indicate T4 spread of the lung cancer. As you know, in ovarian cancer the lavage is included in TNM classification and is included as a suffix to the histologic stage of the tumor. I think that if the result and the procedure are added to the TNM classification it must be the same; we have to add the result as a suffix to the $T$ classification of the tumor because survival is not as poor in the patient with a positive cytologic result as in a patient with a malignant pleural effusion.

Dr. Cannon. When looking for malignant cells within the tumor-free parenchyma of the lung, did you look only in the lobe that had been removed or did you ever look in an adjacent lobe? If so, was there violation across the fissure rather than just direct lymphatic extension within the lobe that you had taken out?

Dr. Buhr. We do the lung parenchyma tissue cultures from the same lobe and not from the other lobe. But the study with our lung tissue cultures was too small to find any statistical differences.

Dr. Alan S. Coulson (Stockton, Calif.). I remember years ago we used to culture cancer cells with lymphocytes to see if the body's immune system would somehow attack the cells in culture. It seems to me you have a perfect model here if you could take some white cells or lymphocytes from the patient and culture the cancer cells with the white cells through 3 or 4 days to see whether there is a reaction that would be predictive of patient survival.

Dr. Buhr. I think the study with the lung tissue cultures is too small to allow any other examples or explanations. I do not believe we find new tumor cells. We find dormant tumor cells in the lung that we have not detected earlier with normal histologic studies, and we detect those tumor cells after incubation and tissue culture. And thank you for your comment. We will try this study with lymphocytes and lung tissue cultures in the future. 